\title{
A novel mutation of the MYH7 gene in a patient with hypertrophic cardiomyopathy
}

\author{
Nicholas Goel ${ }^{1}$, Charles B. Huddleston ${ }^{2}$, Andrew C. Fiore ${ }^{2}$ \\ ${ }^{1}$ Division of Cardiology, Department of Pediatrics, ${ }^{2}$ Division of Cardiothoracic Surgery, Department of Surgery, \\ Saint Louis University School of Medicine, St. Louis, MO, USA. E-mail: nicholas.goel@uphs.upenn.edu \\ Received: 5th February 2017, Accepted: 13th August 2017
}

SUMMARY: Goel N, Huddleston CB, Fiore AC. A novel mutation of the MYH7 gene in a patient with hypertrophic cardiomyopathy. Turk J Pediatr 2018; 60: 315-318.

Hypertrophic cardiomyopathy (HCM) is a genetic disorder characterized by asymmetric cardiac hypertrophy due to inherited mutations in genes that encode sarcomeric proteins. MYH7, which encodes $\beta$-myosin heavy chain, is among the most commonly mutated genes in patients affected by HCM. We aimed to identify the specific mutation responsible for HCM in a sixmonth old Caucasian patient. NextGen DNA sequencing revealed a novel p.Ala1328Thr (A1328T) mutation of MYH7 in the affected patient as well as his asymptomatic father and asymptomatic brother. The clinical details of this mutation are described for the first time in this report. The genetic variant affects a residue that is highly conserved across species. Theoretical analysis suggests that A1328T is very likely deleterious to $\beta$-myosin heavy chain protein structure and function. Furthermore, this novel mutation was not observed with any significant frequency in approximately 6,500 healthy individuals of European and African American ancestry in the NHLBI Exome Sequencing Project, underlining the potential pathogenicity of this variant.

Key words: hypertrophic cardiomyopathy, $\beta$-myosin, MYH7, pediatric cardiology, congenital heart defect.

Hypertrophic cardiomyopathy (HCM) is a primary myocardial disorder characterized by unexplained asymmetric thickening of the interventricular septum and left ventricular free wall.1-3 The prevalence of HCM is 1 in 500 , greater than that of any other monogenic disease of the heart. ${ }^{4}$ Moreover, it is the most common cause of sudden cardiac arrest (SCA) in individuals younger than $35 .{ }^{5} \mathrm{HCM}$ is inherited in an autosomal dominant pattern and exhibits vast genetic heterogeneity, with at least 12 causative genes documented to date, most of which encode cardiac sarcomeric proteins such $\beta$-myosin heavy chain (MYH7), myosin binding protein $\mathrm{C}$ (MYBPC3), cardiac troponin $\mathrm{T}$ (TNNT2), troponin I (TNNI3), cardiac alpha-actin (ACTC), and alpha-tropomyosin (TPM1). ${ }^{6-8}$ Defects at MYH7 are among the most common, accounting for about $40 \%$ of sarcomeric mutations in HCM. ${ }^{9,10}$ In general, mutations in MYH7 tend to cause earlier onset of severe disease and carry higher risk of SCA than other HCM-causing mutations. ${ }^{11-13}$
Nonetheless, phenotypic expression resulting from mutations at MYH7 can be remarkably heterogeneous, with intra- and inter-familial variations ranging from severely malignant to entirely asymptomatic. ${ }^{6}$ Even individuals with identical mutations may exhibit different clinical presentations, suggesting a role of modifier genes and environmental factors. The inherent complexity of HCM already encountered suggests there is still much to be learned about the genetic underpinnings of this disease, making it imperative to explore the genetic backgrounds of HCM patients when possible. Here we report a case of an affected 2-year old boy who is a carrier of a mutation at MYH7 that has yet to be reported in association with HCM.

\section{Case Report}

\section{Clinical presentation}

A 34-year old woman (G2P2) was referred for fetal echocardiography after the detection of enlarged right atrium and right ventricle in the 
fetus on comprehensive obstetric ultrasound in the 32nd week of pregnancy. The fetal cardiac evaluation confirmed dilated right atrium and right ventricle, but no other abnormality was seen.

The patient was born at 38 weeks gestation by vaginal delivery and weighed $3.283 \mathrm{~kg}$ with Apgar scores of 8 and 9. Postnatal echocardiography revealed severe pulmonary hypertension, in addition to the previously noted dilation of the right atrium and right ventricle. The etiology of the pulmonary hypertension was not clear. Persistent neonatal pulmonary hypertension was the presumed diagnosis. The patient received supportive management for pulmonary hypertension in the neonatal intensive care unit. The pulmonary hypertension improved in 3-4 days, and the baby was discharged home with advice to follow up with pediatric cardiology in 3-4 months.

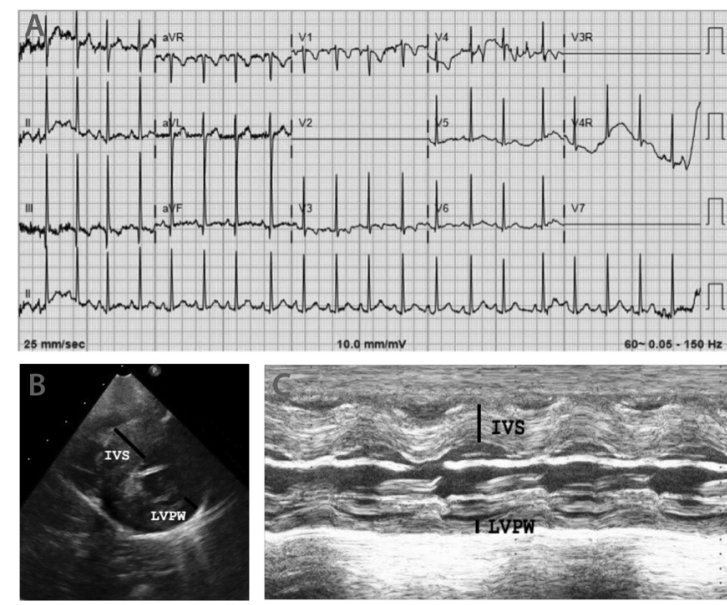

Fig. 1. (A) Standard 12-lead electrocardiogram shows high voltages and $\mathrm{T}$-wave inversion, suggesting possible biventricular hypertrophy. (B, C) Echocardiogram of in parasternal short-axis view (B) and $\mathrm{M}$ mode (C) demonstrate marked asymmetrical thickening of the interventricular septum. IVS, interventricular septum; LVPW, left ventricular posterior wall.
The patient returned for routine cardiac followup at 6 months of age. He had no symptoms at that time. Development and weight gain were normal. An echocardiogram revealed marked asymmetrical septal hypertrophy suggestive of hypertrophic cardiomyopathy (Fig. 1B, Fig. 1C). No sub-aortic obstruction, mitral valve insufficiency, or sub-pulmonary obstruction was seen. His blood pressure was normal. An electrocardiogram revealed possible biventricular hypertrophy (Fig. 1A). A cardiac MRI was performed that revealed marked asymmetrical septal hypertrophy of the atrioventricular septum with no evidence of outflow tract obstruction or myocardial scarring. Storage disorder was considered unlikely. He underwent 24-hour Holter monitor which did not reveal any ventricular or supraventricular arrhythmia. An echocardiogram was performed on the baby's mother, father and only sibling. All three echocardiograms were normal. There was no evidence of hypertrophic cardiomyopathy in any of the family members.

\section{Genetic analysis}

After obtaining informed consent from the parents, blood and buccal cell samples obtained from the patient were submitted to the commercial lab GeneDx (GeneDx, Galthersburg, $\mathrm{MD})$ for genetic analysis at 18 genes previously identified in association with HCM or HCM phenocopies. These were MYH7, TNNT2, MYBPC3, TNNI3, TPM1, ACTC, MYL3, MYL2, CAV3, MTTG, MTTI, MTTK, MTTQ, TNNC1, TTR, PRKAG2, LAMP2 (Danon disease), and GLA (Fabry disease). The coding regions and splice junctions of the 18 genes were enriched using a proprietary targeted capture system developed by GeneDx. These targeted regions were sequenced simultaneously by massively parallel (NextGen) sequencing on an Illumina platform (Illumina, San Diego, CA) with

\begin{tabular}{|lll|}
\hline Homo sapiens & $\mathbf{s p | P 1 2 8 8 3 |}$ & LTRGKLTYTQ QLEDLKRQLE EEVKAKNALA HALQSARHDC DLLREQYEEE \\
Mus musculus & $\mathbf{s p | Q 9 1 2 8 3 |}$ & LTRGKLTYTQ QLEDLKRQLE EEVKAKNALA HALQSARHDC DLLREQYEEE \\
Rattus norvegicus & $\mathbf{s p | P 0 2 5 6 4 |}$ & LTRGKLTYTQ QLEDLKRQLE EEVKAKNALA HALQSARHDC DLLREQYEEE \\
Felis catus & $\mathbf{s p | P 4 9 8 2 4 |}$ & LTRGKLTYTQ QLEDLKRQLE EEVKAKNALA HALQSARHDC DLLREQYEEE \\
Bos taurus & $\mathbf{s p | Q 9 B E 3 9 |}$ & LTRGKLTYTQ QLEDLKRQLE EEVKAKNALA HALQSARHDC DLLREQYEEE \\
Sus scrofa & $\mathbf{s p | P 7 9 2 9 2 |}$ & LTRGKLTYTQ QLEDLKRQLE EEVKAKNALA HALQSARHDC DLLREQYEEE \\
Equus caballus & $\mathbf{s p | Q 8 M U 0 9 |}$ & LTRGKLTYTQ QLEDLKRQLE EEVKAKNALA HALQSARHDC DLLREQYEEE \\
\hline
\end{tabular}

Fig. 2. The Ala 1328 residue of the cardiac MYH7 protein is highly conserved across species. 
paired-end reads. Bi-directional sequence was assembled, aligned to reference gene sequences based on human genome build GRCh37/UCSC hg19, and analyzed for sequence variants. Capillary sequencing was used to confirm all potentially pathogenic variants and to obtain sequence for regions where fewer than 15 reads were achieved by NextGen sequencing.

Sequencing of the MYH7 gene identified a heterozygous $\mathrm{G}$ to $\mathrm{A}$ transition at nucleotide 3982 (p.Ala1328Thr or A1328T). The mutation had not been previously described in association with HCM. Genetic testing was performed on the patient's parents and only sibling. The father and brother were found to have the same mutation as the patient. The mutation was not seen in the mother.

Informed consent was obtained from all individual participants included in the study.

\section{Discussion}

This is the first report providing a detailed clinical presentation of a patient with a mutation, A1328T in MYH7, which has yet to be reported in association with HCM. The A1328T variant was identified once previously in an individual with dilated cardiomyopathy, but was classified as a variant of uncertain significance. ${ }^{14}$ Our analysis aims to lend insight into the potential pathogenecity of this variant. The heterozygous $G$ to $A$ transition at nucleotide 3982 , a position that is conserved across species (Fig. 2), results in the replacement of a non-polar, hydrophobic alanine residue with a polar, hydrophilic threonine residue. The A1328T variant was not observed with any significant frequency in approximately 6,500 healthy individuals of European and African American ancestry in the NHLBI Exome Sequencing Project (evs. gs.washington.edu/EVS), underlining the potential pathogenic nature of this mutation. PolyPhen-2 (http://genetics.bwh.harvard.edu/ pph2/), a program for predicting the potential impact of an amino acid substitution on the structure and function of a human protein, was used to evaluate the A1328T mutation. The resulting protein function score was 0.988 , classified as "probably damaging" (benign: 0.00-0.20, possibly damaging: $0.20-0.85$, probably damaging: 0.85-1.00). Furthermore, pathogenic variants in nearby residues (N1327K,
A1332T) have been reported in the Human Gene Mutation Database in association with cardiomyopathy, supporting the functional importance of this region of the protein. ${ }^{15}$ The effect of the N1327T mutation, in particular, on the structure and function of the beta-cardiac myosin protein has been shown to be more severe than the effects of other HCM-causing mutations at MYH7.16

In line with previous evidence on the great variability of phenotypic expression of the disease state in families affected by HCM8, we found that the presence of the responsible genetic mutation was not always associated with cardiac hypertrophy in this family. While the affected son was diagnosed with HCM at 6 months of age, the father and brother, both of whom also possessed the mutant gene, had normal echocardiograms at last evaluation (age $36 \mathrm{yr}$ and $5 \mathrm{yr}$, respectively). This phenomenon has been correlated to the presence of modifier genes that may play a role in the phenotypic expression of cardiac hypertrophy in HCM. ${ }^{17,18}$

In conclusion, we describe a MYH7 gene mutation that has yet to be reported in a patient affected by HCM. The absence of the mutation from a control population and its theoretical effects on the physical properties of the coded protein suggest potential pathogenicity. Further studies are needed to better elucidate the role of this mutation in the development of HCM.

\section{Acknowledgements}

The authors thank the family for their participation in this study.

\section{REFERENCES}

1. Elliott P, McKenna WJ. Hypertrophic cardiomyopathy. Lancet 2004; 363: 1881-1891.

2. Maron BJ. Hypertrophic cardiomyopathy: A systematic review. JAMA 2002; 287: 1308-1320.

3. Maron BJ, McKenna WJ, Danielson GK, et al; Task Force on Clinical Expert Consensus Documents. American College of Cardiology; Committee for Practice Guidelines. European Society of Cardiology. American College of Cardiology/European Society of Cardiology clinical expert consensus document on hypertrophic cardiomyopathy. A report of the American College of Cardiology Foundation Task Force on Clinical Expert Consensus Documents and the European Society of Cardiology Committee for Practice Guidelines. J Am Coll Cardiol 2003; 42: 1687-1713.

4. Maron BJ, Gardin JM, Flack JM, Gidding SS Kurosaki TT, Bild DE. Prevalence of hypertrophic cardiomyopathy in a general population of young 
adults. Echocardiographic analysis of 4111 subjects in the CARDIA Study. Coronary Artery Risk Development in (Young) Adults. Circulation 1995; 92: 785-789.

5. Maron BJ, Doerer JJ, Haas TS, Tierney DM, Mueller FO. Sudden deaths in young competitive athletes: Analysis of 1866 deaths in the United States, 19802006. Circulation 2009; 119: 1085-1092.

6. Richard P, Charron P, Carrier L, et al; EUROGENE Heart Failure Project. Hypertrophic cardiomyopathy: Distribution of disease genes, spectrum of mutations, and implications for a molecular diagnosis strategy. Circulation 2003; 107: 2227-2232.

7. Kelly BP, Russell MW, Hennessy JR, Ensing GJ. Severe hypertrophic cardiomyopathy in an infant with a novel PRKAG2 gene mutation: Potential differences between infantile and adult onset presentation. Pediatr Cardiol 2009; 30: 1176-1179.

8. Maron BJ, Maron MS, Semsarian C. Genetics of hypertrophic cardiomyopathy after 20 years: Clinical perspectives. J Am Coll Cardiol 2012; 60: 705-715.

9. Maron BJ. The 2009 international hypertrophic cardiomyopathy summit. Am J Cardiol 2010; 105: 1164-1168.

10. McNally EM. Beta-myosin heavy chain gene mutations in familial hypertrophic cardiomyopathy: The usual suspect? Circ Res 2002; 90: 246-247.

11. Arad M, Seidman JG, Seidman CE. Phenotypic diversity in hypertrophic cardiomyopathy. Hum Mol Genet 2002; 11: 2499-2506.

12. Watkins H, Rosenzweig A, Hwang DS, et al. Characteristics and prognostic implications of myosin missense mutations in familial hypertrophic cardiomyopathy. N Engl J Med 1992; 326: 1108-1114.
13. Epstein ND, Cohn GM, Cyran F, Fananapazir L. Differences in clinical expression of hypertrophic cardiomyopathy associated with two distinct mutations in the beta-myosin heavy chain gene. A 908Leu-Val mutation and a 403Arg-Gln mutation. Circulation 1992; 86: 345-352.

14. Pugh TJ, Kelly MA, Gowrisankar S, et al. The landscape of genetic variation in dilated cardiomyopathy as surveyed by clinical DNA sequencing. Genet Med 2014; 16: 601-608.

15. Stenson PD, Mort M, Ball EV, Shaw K, Phillips A, Cooper DN. The Human Gene Mutation Database: Building a comprehensive mutation repository for clinical and molecular genetics, diagnostic testing and personalized genomic medicine. Hum Genet 2014; 133: $1-9$.

16. Wolny M, Colegrave M, Colman L, White E, Knight PJ, Peckham M. Cardiomyopathy mutations in the tail of $\beta$ cardiac myosin modify the coiled-coil structure and affect integration into thick filaments in muscle sarcomeres in adult cardiomyocytes. J Biol Chem 2013; 288: 31952-31962.

17. Daw EW, Chen SN, Czernuszewicz G, et al. Genomewide mapping of modifier chromosomal loci for human hypertrophic cardiomyopathy. Hum Mol Genet 2007; 16: 2463-2471.

18. Su M, Wang J, Kang L, et al. Rare variants in genes encoding MuRF1 and MuRF2 are modifiers of hypertrophic cardiomyopathy. Int J Mol Sci 2014; 15: 9302-9313. 